\title{
An Approximate Expression for the Average AoI in a Multi-Source M/G/1 Queueing Model
}

\author{
Mohammad Moltafet and Markus Leinonen \\ Centre for Wireless Communications - Radio Technologies \\ University of Oulu, Finland \\ e-mail: \{mohammad.moltafet, markus.leinonen\}@oulu.fi
}

\author{
Marian Codreanu \\ Department of Science and Technology \\ Linköping University, Sweden \\ e-mail: marian.codreanu@liu.se
}

\begin{abstract}
Freshness of status update packets is essential for wide range of real-time Internet of things applications. In this paper, we study the information freshness of a single-server multi-source queueing model under a first-come first-served (FCFS) serving policy. In the considered model, each source independently generates status update packets according to a Poisson process. The information freshness of the status updates of each source is evaluated by the average age of information (AoI). We derive an approximate expression for the average AoI for a multi-source $M / G / 1$ queueing model having a general service time distribution. Simulation results are provided to validate and assess the tightness of the proposed approximate expression for the average AoI in the M/G/1 queueing model where the service time follows a gamma distribution.
\end{abstract}

\section{INTRODUCTION}

With the advent of new services in 5G and beyond such as real-time Internet of things applications, autonomous flying vehicles, and cyber physical applications, the delivery of fresh status updates is gaining increasing interest. For enabling these services, low power sensors are assigned to send status updates about a random process to intended destinations [1]-[5]. Such a status update system can monitor, e.g., temperature of a specific environment [1], and a vehicular status (position, acceleration, etc.) [6]. Freshness of the sensors' information at the destination is vital for the services. Recently, the age of information (AoI) was proposed as a destination-centric metric to measure the information freshness in status update systems [7], [8]. A status update packet contains the measured value of a monitored process and a time stamp representing the time when the sample was generated. Due to wireless channel access, channel errors, and fading etc., communicating a status update packet through the network experiences a random delay. If at a time instant $t$, the most recently received status update packet contains the time stamp $U(t)$, AoI is defined as the random process $\Delta(t)=t-U(t)$. Thus, the AoI measures for each sensor the time elapsed since the last received status update packet was generated at the sensor.

The first queueing theoretic work on AoI is [7] where the authors derived the average AoI for a single-source M/M/1 first-come first-served (FCFS) queueing model. The work [9] was the first to investigate the average AoI in a multisource setup. The authors of [9] derived the average AoI for a multi-source M/M/1 FCFS queueing model. The closedform expressions for the average AoI and average peak AoI in a multi-source $\mathrm{M} / \mathrm{G} / 1 / 1$ preemptive queueing model were derived in [10]. The authors of [11] studied the average AoI in a multi-source $\mathrm{M} / \mathrm{G} / 1$ queueing model under the FCFS policy. However, they wrongly used steady-state properties of a queueing system in calculating the average AoI.

In this paper, we analyze the average AoI of the different sources in a single-server multi-source queueing model under an FCFS service policy with Poisson packet arrivals. We point out the difficulties in deriving the average AoI for an M/G/1 case. Consequently, as the main contribution of this paper, we derive an approximate expression for the average AoI in a multi-source M/G/1 queueing model. Simulation results show that the proposed approximation is relatively tight in the $\mathrm{M} / \mathrm{G} / 1$ case where the service time follows a gamma distribution.

\section{System Model And Definitions}

We consider a system consisting of a set of independent sources denoted by $\mathcal{C}=\{1, \ldots, C\}$ and one server. Each source observes a random process, representing, e.g., temperature, vehicular speed or location at random time instants. A remote destination is interested in timely information about the status of these random processes. We assume that the status update packets of source $c$ are generated according to the Poisson process with rate $\lambda_{c}, c \in \mathcal{C}$.

Definition 1 (AoI). Let $t_{c, i}$ denote the time instant at which the $i$ th packet of source $c$ was generated, and $t_{c, i}^{\prime}$ denote the time instant at which this packet arrives at the destination. At a time instant $\tau$, the index of the most recently received packet of source $c$ is given by $N_{c}(\tau)=\max \left\{i^{\prime} \mid t_{c, i^{\prime}}^{\prime} \leq \tau\right\}$, and the time stamp of the most recently received packet of source $c$ is $U_{c}(\tau)=t_{c, N_{c}(\tau)}$. The AoI of source $c$ at the destination is defined as the random process $\Delta_{c}(t)=t-U_{c}(t)$.

From here onwards, we refer to the $i$ th packet from source $c$ simply as packet $c, i$. Let $X_{c, i}$ denote the $i$ th interarrival time of source $c ; W_{c, i}$ denote the waiting time of packet $c, i$; $S_{c, i}$ denote the service time of packet $c, i$; and $T_{c, i}$ denote the system time of packet $c, i$, i.e., the time interval the packet spends in the system which consists of the sum of the waiting time and the service time, $T_{c, i}=S_{c, i}+W_{c, i}$. Then, the average AoI of source $c$, denoted as $\Delta_{c}$, is given as [7]

$$
\begin{aligned}
\Delta_{c} & =\lambda_{c}\left(\frac{\mathbb{E}\left[X_{c, i}^{2}\right]}{2}+\mathbb{E}\left[X_{c, i} T_{c, i}\right]\right) \\
& =\lambda_{c}\left(\mathbb{E}\left[X_{c, i}^{2}\right] / 2+\mathbb{E}\left[X_{c, i}\left(S_{c, i}+W_{c, i}\right)\right]\right) .
\end{aligned}
$$




\section{Average AoI in a Multi-Source M/G/1 Queueing Model}

To evaluate the AoI of one source, we can consider two sources without loss of generality. Thus, we evaluate the AoI of source 1 by aggregating the other $C-1$ sources into source 2 having the Poisson arrival rate $\lambda_{2}=\sum_{c^{\prime} \in \mathcal{C} \backslash\{c\}} \lambda_{c^{\prime}}$. The mean service time for each packet in the system is equal, given as $\mathbb{E}\left[S_{1, i}\right]=\mathbb{E}\left[S_{2, i}\right]=1 / \mu, \forall i$. Let $\rho_{1}=\lambda_{1} / \mu$ and $\rho_{2}=\lambda_{2} / \mu$ be the load of source 1 and 2 , respectively. Since packets of each source are generated according to the Poisson process and the sources are independent, the packet generation in the system follows the Poisson process with rate $\lambda=\lambda_{1}+\lambda_{2}$, and the overall load in the system is $\rho=\rho_{1}+\rho_{2}=\lambda / \mu$. Since we do not assume any specific probability density function (PDF) for the service time, the considered model is referred to as a multi-source M/G/1 queueing model.

In the following, we derive the average AoI (1) for source 1 , denoted as $\Delta_{1}$. The first term in (1) is easy to compute. Namely, since the interarrival time of source 1 follows the exponential distribution with parameter $\lambda_{1}$, we have $\mathbb{E}\left[X_{1, i}^{2}\right]=$ $2 / \lambda_{1}^{2}$. However, because the random variables $X_{1, i}$ and $T_{1, i}$ are dependent, the most challenging part in calculating (1) is $\mathbb{E}\left[X_{1, i} T_{1, i}\right]=\mathbb{E}\left[X_{1, i}\left(W_{1, i}+S_{1, i}\right)\right]$ which is derived next.

Since the interarrival time and service time of the packet $1, i$ are independent, we have

$$
\mathbb{E}\left[X_{1, i}\left(W_{1, i}+S_{1, i}\right)\right]=\mathbb{E}\left[X_{1, i} W_{1, i}\right]+\frac{\mathbb{E}\left[X_{1, i}\right]}{\mu},
$$

Next, we derive $\mathbb{E}\left[X_{1, i} W_{1, i}\right]$. To calculate $\mathbb{E}\left[X_{1, i} W_{1, i}\right]$, we follow the approach of [9] and characterize the waiting time $W_{1, i}$ by means of two events $E_{1, i}^{\mathrm{B}}$ and $E_{1, i}^{\mathrm{L}}$ as

$$
E_{1, i}^{\mathrm{B}}=\left\{T_{1, i-1} \geq X_{1, i}\right\}, \quad E_{1, i}^{\mathrm{L}}=\left\{T_{1, i-1}<X_{1, i}\right\} .
$$

Here, brief event $E_{1, i}^{\mathrm{B}}$ is the event where the interarrival time of packet $1, i$ is brief, i.e., the interarrival time of packet $1, i$ is shorter than the system time of packet $1, i-1$. On the contrary, long event $E_{1, i}^{\mathrm{L}}$ refers to the complementary event where the interarrival time of packet $1, i$ is long, i.e., the interarrival time of packet $1, i$ is longer than the system time of packet $1, i-1$.

Under the event $E_{1, i}^{\mathrm{B}}$, the waiting time of packet $1, i\left(W_{1, i}\right)$ contains two terms: 1 ) the residual system time to complete serving packet $1, i-1$, and 2 ) the sum of service times of the source 2 packets that arrived during $X_{1, i}$ and must be served before packet $1, i$ according to the FCFS policy. Under the event $E_{1, i}^{\mathrm{L}}$, the waiting time of packet $1, i$ contains two terms: 1) the possible residual service time of a source 2 packet that is under service at the arrival instant of packet $1, i$, and 2) the sum of service times of source 2 packets in the queue that must be served before packet $1, i$ according to the FCFS policy. Thus, by means of the two events in (3), the waiting time for packet $1, i$ can be expressed as

$$
W_{1, i}= \begin{cases}T_{1, i-1}-X_{1, i}+\sum_{i^{\prime} \in \mathcal{M}_{2, i}^{\mathrm{B}}} S_{2, i^{\prime}}, & E_{1, i}^{\mathrm{B}} \\ \sum_{i^{\prime} \in \mathcal{M}_{2, i}^{\mathrm{L}}} S_{2, i^{\prime}}+R_{2, i}^{\mathrm{L}}, & E_{1, i}^{\mathrm{L}},\end{cases}
$$

where $\mathcal{M}_{2, i}^{\mathrm{B}}$ is the set of indices of queued packets of source
2 that must be served before packet $1, i$ under the event $E_{1, i}^{\mathrm{B}}$, where $\left|\mathcal{M}_{2, i}^{\mathrm{B}}\right|=M_{2, i}^{\mathrm{B}} ; \mathcal{M}_{2, i}^{\mathrm{L}}$ is the set of indices of source 2 packets that are in the queue (but not under service) at the arrival instant of packet $1, i$ conditioned on the event $E_{1, i}^{\mathrm{L}}$ and, thus, must be served before packet $1, i$, where $\left|\mathcal{M}_{2, i}^{\mathrm{L}}\right|=M_{2, i}^{\mathrm{L}}$; $R_{2, i}^{\mathrm{L}}$ is a random variable that represents the possible residual service time of the packet of source 2 that is under service at the arrival instant of packet $1, i$ conditioned on the event $E_{1, i}^{\mathrm{L}}$.

For the case $E_{1, i}^{\mathrm{B}}$, let us further divide the waiting time $W_{1, i}$ in (4) into two terms $R_{1, i}^{\mathrm{B}}$ and $S_{1, i}^{\mathrm{B}}$ as follows. Let

$$
R_{1, i}^{\mathrm{B}}=T_{1, i-1}-X_{1, i}
$$

represent the residual system time to complete serving packet $1, i-1$ and let

$$
S_{1, i}^{\mathrm{B}}=\sum_{i^{\prime} \in \mathcal{M}_{2, i}^{\mathrm{B}}} S_{2, i^{\prime}}
$$

represent the sum of service times of source 2 packets that arrived during $X_{1, i}$ and must be served before packet $1, i$. Similarly for the event $E_{1, i}^{\mathrm{L}}$, let

$$
S_{1, i}^{\mathrm{L}}=\sum_{i^{\prime} \in \mathcal{M}_{2, i}^{\mathrm{L}}} S_{2, i^{\prime}}
$$

represent the sum of service times of source 2 packets that must be served before packet 1, $i$. Based on (5), (6), and (7), $\mathbb{E}\left[X_{1, i} W_{1, i}\right]$ in (2) can be expressed as

$$
\begin{aligned}
\mathbb{E}\left[X_{1, i} W_{1, i}\right]= & \left(\mathbb{E}\left[R_{1, i}^{\mathrm{B}} X_{1, i} \mid E_{1, i}^{\mathrm{B}}\right]+\mathbb{E}\left[S_{1, i}^{\mathrm{B}} X_{1, i} \mid E_{1, i}^{\mathrm{B}}\right]\right) P\left(E_{1, i}^{\mathrm{B}}\right) \\
& +\mathbb{E}\left[\left(S_{1, i}^{\mathrm{L}}+R_{2, i}^{\mathrm{L}}\right) X_{1, i} \mid E_{1, i}^{\mathrm{L}}\right] P\left(E_{1, i}^{\mathrm{L}}\right),
\end{aligned}
$$

where $P\left(E_{1, i}^{\mathrm{B}}\right)$ and $P\left(E_{1, i}^{\mathrm{L}}\right)$ denote the probabilities of the events $E_{1, i}^{\mathrm{B}}$ and $E_{1, i}^{\mathrm{L}}$, respectively.

Next, we derive the expressions for $P\left(E_{1, i}^{\mathrm{B}}\right)$ and $P\left(E_{1, i}^{\mathrm{L}}\right)$ in (8). Then, by referring to $\mathbb{E}\left[R_{1, i}^{\mathrm{B}} X_{1, i} \mid E_{1, i}^{\mathrm{B}}\right], \mathbb{E}\left[S_{1, i}^{\mathrm{B}} X_{1, i} \mid E_{1, i}^{\mathrm{B}}\right]$, and $\mathbb{E}\left[\left(S_{1, i}^{\mathrm{L}}+R_{2, i}^{\mathrm{L}}\right) X_{1, i} \mid E_{1, i}^{\mathrm{L}}\right]$ in (8) as the first, the second, and the third conditional expectation terms of (8), we derive the first and second terms in Sections III-1 and III-2, respectively, and in Section III-3 we point out the difficulties in computing the third term for a generic service time distribution.

Lemma 1. The probabilities of the events $E_{1, i}^{\mathrm{B}}$ and $E_{1, i}^{\mathrm{L}}$ in (3) are calculated as follows:

$$
\begin{aligned}
& P\left(E_{1, i}^{\mathrm{B}}\right)=\frac{L_{S}\left(\lambda_{1}\right)\left(\lambda+(\rho-1) \lambda_{1}\right)-\lambda_{2}}{\lambda L_{S}\left(\lambda_{1}\right)-\lambda_{2}}, \\
& P\left(E_{1, i}^{\mathrm{L}}\right)=\frac{(1-\rho) \lambda_{1} L_{S}\left(\lambda_{1}\right)}{\lambda L_{S}\left(\lambda_{1}\right)-\lambda_{2}},
\end{aligned}
$$

where $L_{S}\left(\lambda_{1}\right)=\mathbb{E}\left[e^{-\lambda_{1} S}\right]$ is the Laplace transform of the PDF of the service time $S$ at $\lambda_{1}$; note that the service times of all packets are stochastically identical as $S_{1, i}={ }^{\mathrm{st}} S_{2, i}={ }^{\mathrm{st}} S, \forall i$.

Proof. Using the facts that $T_{1, i-1}$ and $X_{1, i}$ are independent and the PDF of $X_{1, i}$ is $f_{X_{1, i}}(x)=\lambda_{1} e^{-\lambda_{1} x}, P\left(E_{1, i}^{\mathrm{B}}\right)$ can be written as

$$
\begin{aligned}
& P\left(E_{1, i}^{\mathrm{B}}\right)=\int_{0}^{\infty} P\left(T_{1, i-1} \geq X_{1, i} \mid T_{1, i-1}=t\right) f_{T_{1, i-1}}(t) \mathrm{d} t \\
& =\int_{0}^{\infty} F_{X_{1, i}}(t) f_{T_{1, i-1}}(t) \mathrm{d} t=1-\int_{0}^{\infty} e^{-\lambda_{1} t} f_{T_{1, i-1}}(t) \mathrm{d} t
\end{aligned}
$$




$$
\stackrel{(a)}{=} 1-\mathbb{E}\left[e^{-\lambda_{1} T}\right]=1-L_{T}\left(\lambda_{1}\right),
$$

where equality (a) follows because the system times of different packets are stochastically identical, i.e., $T_{1, i}={ }^{\text {st }} T_{2, i}={ }^{\text {st }}$ $T, \forall i$ [9], [12]; $F_{X_{1, i}}(x)$ is the cumulative distribution function (CDF) of $X_{1, i}$; and $L_{T}\left(\lambda_{1}\right)$ denotes the Laplace transform of the PDF of the system time $T$ at $\lambda_{1}$. Because $E_{1, i}^{\mathrm{L}}$ is the complementary event of $E_{1, i}^{\mathrm{B}}$, we have

$$
P\left(E_{1, i}^{\mathrm{L}}\right)=1-P\left(E_{1, i}^{\mathrm{B}}\right)=L_{T}\left(\lambda_{1}\right) .
$$

The relation between the Laplace transforms of the PDFs of the system time $T$ and service time $S$ is given as [13, Sect. 5.1.2]

$$
L_{T}(a)=\frac{(1-\rho) a L_{S}(a)}{a-\lambda\left(1-L_{S}(a)\right)} \triangleq H_{S}(a) .
$$

Finally, substituting (12) in (10) and (11) results in the expressions in (9).

1) The First Conditional Expectation in (8): Let us now focus on the first conditional expectation term $\mathbb{E}\left[R_{1, i}^{\mathrm{B}} X_{1, i} \mid E_{1, i}^{\mathrm{B}}\right]$ in (8). According to (5), this term is expressed as follows:

$$
\begin{aligned}
& \mathbb{E}\left[R_{1, i}^{\mathrm{B}} X_{1, i} \mid E_{1, i}^{\mathrm{B}}\right]=\mathbb{E}\left[T_{1, i-1} X_{1, i} \mid E_{1, i}^{\mathrm{B}}\right]-\mathbb{E}\left[X_{1, i}^{2} \mid E_{1, i}^{\mathrm{B}}\right] \\
& =\int_{0}^{\infty} \int_{0}^{\infty} x t f_{X_{1, i}, T_{1, i-1} \mid E_{1, i}^{\mathrm{B}}}(x, t) \mathrm{d} x \mathrm{~d} t-\int_{0}^{\infty} x^{2} f_{X_{1, i} \mid E_{1, i}^{\mathrm{B}}}(x) \mathrm{d} x,
\end{aligned}
$$

where $f_{X_{1, i} \mid E_{1, i}^{\mathrm{B}}}(x)$ is the conditional PDF of the interarrival time $X_{1, i}$ given the event $E_{1, i}^{\mathrm{B}}$ and $f_{X_{1, i}, T_{1, i-1} \mid E_{1, i}^{\mathrm{B}}}(x, t)$ is the conditional joint PDF of the interarrival time $X_{1, i}$ and system time $T_{1, i-1}$ given the event $E_{1, i}^{\mathrm{B}}$. They are given by the following two lemmas.

Lemma 2. The conditional PDF $f_{X_{1, i} \mid E_{1, i}^{\mathrm{B}}}(x)$ is given by

$$
f_{X_{1, i} \mid E_{1, i}^{\mathrm{B}}}(x)=\frac{\lambda_{1} e^{-\lambda_{1} x}\left(1-F_{T_{1, i-1}}(x)\right)}{P\left(E_{1, i}^{\mathrm{B}}\right)} .
$$

Due to the space limitations, the proof is given in [14].

Lemma 3. The PDF $f_{X_{1, i}, T_{1, i-1} \mid E_{1, i}^{\mathrm{B}}}(x, t)$ is given by

$f_{X_{1, i}, T_{1, i-1} \mid E_{1, i}^{\mathrm{B}}}(x, t)=\frac{1}{P\left(E_{1, i}^{\mathrm{B}}\right)} \begin{cases}0 & x>t \\ \lambda_{1} e^{-\lambda_{1} x} f_{T_{1, i-1}}(t) & x \leq t .\end{cases}$

Due to the space limitations, the proof is given in [14].

Now, having introduced the conditional PDFs in Lemma 2 and Lemma 3 , we can compute the conditional expectation $\mathbb{E}\left[R_{1, i}^{\mathrm{B}} X_{1, i} \mid E_{1, i}^{\mathrm{B}}\right]$ in (13). Using Lemma 3, the first term in (13) is calculated as

$$
\begin{aligned}
& \mathbb{E}\left[T_{1, i-1} X_{1, i} \mid E_{1, i}^{\mathrm{B}}\right]=\int_{0}^{\infty} \int_{0}^{\infty} x t f_{X_{1, i}, T_{1, i-1} \mid E_{1, i}^{\mathrm{B}}}(x, t) \mathrm{d} x \mathrm{~d} t \\
& =\frac{1}{P\left(E_{1, i}^{\mathrm{B}}\right)} \int_{0}^{\infty} \int_{0}^{t} t x \lambda_{1} e^{-\lambda_{1} x} f_{T_{1, i-1}}(t) \mathrm{d} x \mathrm{~d} t \\
& =\frac{1}{P\left(E_{1, i}^{\mathrm{B}}\right)}\left(-\mathbb{E}\left[T^{2} e^{-\lambda_{1} T}\right]-\frac{\mathbb{E}\left[T e^{-\lambda_{1} T}\right]}{\lambda_{1}}+\frac{\mathbb{E}[T]}{\lambda_{1}}\right) \\
& \stackrel{(a)}{=} \frac{1}{P\left(E_{1, i}^{\mathrm{B}}\right)}\left(-L_{T}^{\prime \prime}\left(\lambda_{1}\right)+\frac{L_{T}^{\prime}\left(\lambda_{1}\right)}{\lambda_{1}}+\frac{\mathbb{E}[W]+1 / \mu}{\lambda_{1}}\right),
\end{aligned}
$$

where in equality (a) the first and second derivative of the Laplace transform, $L_{T}^{\prime}$ and $L_{T}^{\prime \prime}$ at $\lambda_{1}$, respectively, were obtained using the feature of the Laplace transform that for any function $f(y), y \geq 0$, we have [15, Sect. 13.5]

$$
L_{y^{n} f(y)}(a)=(-1)^{n} \frac{\mathrm{d}^{n}\left(L_{f(y)}(a)\right)}{\mathrm{d} a^{n}} .
$$

Following the notation in (12) as $L_{T}^{\prime}(a)=H_{S}^{\prime}(a)$ and $L_{T}^{\prime \prime}(a)=H_{S}^{\prime \prime}(a),(15)$ can be written as

$$
\begin{aligned}
& \mathbb{E}\left[T_{1, i-1} X_{1, i} \mid E_{1, i}^{\mathrm{B}}\right]= \\
& \frac{1}{P\left(E_{1, i}^{\mathrm{B}}\right)}\left(-H_{S}^{\prime \prime}\left(\lambda_{1}\right)+\frac{H_{S}^{\prime}\left(\lambda_{1}\right)+\mathbb{E}[W]+1 / \mu}{\lambda_{1}}\right) .
\end{aligned}
$$

Using Lemma 2, the second term $\mathbb{E}\left[X_{1, i}^{2} \mid E_{1, i}^{\mathrm{B}}\right]$ in (13) is calculated as

$$
\begin{aligned}
\mathbb{E}\left[X_{1, i}^{2} \mid E_{1, i}^{\mathrm{B}}\right] & =\int_{0}^{\infty} x^{2} f_{X_{1, i} \mid E_{1, i}^{\mathrm{B}}}(x) \mathrm{d} x \\
& =\frac{1}{P\left(E_{1, i}^{\mathrm{B}}\right)} \int_{0}^{\infty} x^{2} \lambda_{1} e^{-\lambda_{1} x}\left(1-F_{T_{1, i-1}}(x)\right) \mathrm{d} x \\
& =\frac{1}{P\left(E_{1, i}^{\mathrm{B}}\right)}\left(\frac{2}{\lambda_{1}^{2}}-\lambda_{1} L_{x^{2} F_{T_{1}}(x)}\left(\lambda_{1}\right)\right) .
\end{aligned}
$$

The Laplace transform $L_{x^{2} F_{T_{1}}(x)}\left(\lambda_{1}\right)$ in (18) is given by the following lemma.

Lemma 4. $L_{x^{2} F_{T_{1}}(x)}\left(\lambda_{1}\right)$ is given as follows:

$$
\left.L_{x^{2} F_{T_{1}}(x)}(a)\right|_{a=\lambda_{1}}=\frac{\lambda_{1} H_{S}^{\prime \prime}\left(\lambda_{1}\right)-2 H_{S}^{\prime}\left(\lambda_{1}\right)}{\lambda_{1}^{2}}+\frac{2 H_{S}\left(\lambda_{1}\right)}{\lambda_{1}^{3}} .
$$

Due to the space limitations, the proof is given in [14]. Applying Lemma 4, the conditional expectation in (18) is given as

$$
\begin{aligned}
& \mathbb{E}\left[X_{1, i}^{2} \mid E_{1, i}^{\mathrm{B}}\right]= \\
& \frac{1}{P\left(E_{1, i}^{\mathrm{B}}\right)}\left(\frac{2}{\lambda_{1}^{2}}-H_{S}^{\prime \prime}\left(\lambda_{1}\right)+\frac{2 H_{S}^{\prime}\left(\lambda_{1}\right)}{\lambda_{1}}-\frac{2 H_{S}\left(\lambda_{1}\right)}{\lambda_{1}^{2}}\right) .
\end{aligned}
$$

Finally, substituting (17) and (20) in (13), the first conditional expectation $\mathbb{E}\left[R_{1, i}^{\mathrm{B}} X_{1, i} \mid E_{1, i}^{\mathrm{B}}\right]$ in (8) is given by

$$
\begin{aligned}
& \mathbb{E}\left[R_{1, i}^{\mathrm{B}} X_{1, i} \mid E_{1, i}^{\mathrm{B}}\right]= \\
& \frac{1}{P\left(E_{1, i}^{\mathrm{B}}\right)}\left(\frac{\mathbb{E}[W]+1 / \mu}{\lambda_{1}}-\frac{H_{S}^{\prime}\left(\lambda_{1}\right)}{\lambda_{1}}+\frac{2 H_{S}\left(\lambda_{1}\right)}{\lambda_{1}^{2}}-\frac{2}{\lambda_{1}^{2}}\right) .
\end{aligned}
$$

2) The Second Conditional Expectation in (8): Next, we derive the second term $\mathbb{E}\left[S_{1, i}^{\mathrm{B}} X_{1, i} \mid E_{1, i}^{\mathrm{B}}\right]$ in (8). First, let us elaborate the quantity $M_{2, i}^{\mathrm{B}}$ which is an integral part of calculating (8). Recall that $M_{2, i}^{\mathrm{B}}$ is defined as the number of queued packets of source 2 that must be served before packet $1, i$ according to the FCFS policy under the event $E_{1, i}^{\mathrm{B}}=\left\{T_{1, i-1} \geq X_{1, i}\right\}$. Thus, $M_{2, i}^{\mathrm{B}}$ is equal to the number of arrived (and thus, queued) packets of source 2 during the (brief) interarrival time $X_{1, i}$. Consequently, we have a Markov chain $T_{1, i-1} \leftrightarrow X_{1, i} \leftrightarrow M_{2, i}^{\mathrm{B}}$ conditioned on the event $E_{1, i}^{\mathrm{B}}$, i.e., $M_{2, i}^{\mathrm{B}}$ is independent of $T_{1, i-1}$ given $X_{1, i}$ under the event 
$E_{1, i}^{\mathrm{B}}$. Accordingly, $\mathbb{E}\left[S_{1, i}^{\mathrm{B}} X_{1, i} \mid E_{1, i}^{\mathrm{B}}\right]$ in (8) can be expressed as $\mathbb{E}\left[S_{1, i}^{\mathrm{B}} X_{1, i} \mid E_{1, i}^{\mathrm{B}}\right]=\int_{0}^{\infty} x \mathbb{E}\left[\sum_{i^{\prime} \in \mathcal{M}_{2, i}^{\mathrm{B}}} S_{2, i^{\prime}} \mid E_{1, i}^{\mathrm{B}}, X_{1, i}\right] f_{X_{1, i} \mid E_{1, i}^{\mathrm{B}}} \mathrm{d} x$ $\stackrel{(a)}{=} \frac{1}{\mu} \int_{0}^{\infty} x \mathbb{E}\left[M_{2, i}^{\mathrm{B}} \mid X_{1, i}=x\right] f_{X_{1, i} \mid E_{1, i}^{\mathrm{B}}}(x) \mathrm{d} x$

$\stackrel{(b)}{=} \frac{\rho_{2}}{P\left(E_{1, i}^{\mathrm{B}}\right)} \int_{0}^{\infty} x^{2} \lambda_{1} e^{-\lambda_{1} x}\left(1-F_{T_{1, i-1}}(x)\right) \mathrm{d} x$

$\stackrel{(c)}{=} \frac{\rho_{2}}{P\left(E_{1, i}^{\mathrm{B}}\right)}\left(\frac{2}{\lambda_{1}^{2}}-H_{S}^{\prime \prime}\left(\lambda_{1}\right)+\frac{2 H_{S}^{\prime}\left(\lambda_{1}\right)}{\lambda_{1}}-\frac{2 H_{S}\left(\lambda_{1}\right)}{\lambda_{1}^{2}}\right)$,

where equality (a) follows because (i) the service time $S_{2, i^{\prime}}$ is independent of all other random variables in the system and (ii) by the Markov chain property $T_{1, i-1} \leftrightarrow X_{1, i} \leftrightarrow M_{2, i}^{\mathrm{B}}$ conditioned on $E_{1, i}^{\mathrm{B}}, M_{2, i}^{\mathrm{B}}$ is independent of $T_{1, i-1}$ given $X_{1, i}=x$ under the event $E_{1, i}^{\mathrm{B}}$; equality (b) comes from Lemma 2 and the fact that $\mathbb{E}\left[M_{2, i}^{\mathrm{B}} \mid X_{1, i}=x\right]=\lambda_{2} x$; equality (c) comes from Lemma 4.

3) The Third Conditional Expectation in (8): The third term $\mathbb{E}\left[\left(S_{1, i}^{\mathrm{L}}+R_{2, i}^{\mathrm{L}}\right) X_{1, i} \mid E_{1, i}^{\mathrm{L}}\right]$ in (8) can be calculated as

$$
\begin{gathered}
\mathbb{E}\left[\left(S_{1, i}^{\mathrm{L}}+R_{2, i}^{\mathrm{L}}\right) X_{1, i} \mid E_{1, i}^{\mathrm{L}}\right]=\int_{0}^{\infty} \int_{0}^{\infty} x \mathbb{E}\left[\sum_{i^{\prime} \in \mathcal{M}_{2, i}^{\mathrm{L}}, S_{2, i^{\prime}} \mid \cdots}\right. \\
\left.X_{1, i}=x, T_{1, i-1}=t, E_{1, i}^{\mathrm{L}}\right] f_{X_{1, i} T_{1, i-1} \mid E_{1, i}^{\mathrm{L}}}(x, t) \mathrm{d} x \mathrm{~d} t \\
+\int_{0}^{\infty} \int_{0}^{\infty} x \mathbb{E}\left[R_{2, i}^{\mathrm{L}} \mid X_{1, i}=x, T_{1, i-1}=t, E_{1, i}^{\mathrm{L}}\right] \cdots \\
f_{X_{1, i} T_{1, i-1} \mid E_{1, i}^{\mathrm{L}}}(x, t) \mathrm{d} x \mathrm{~d} t,
\end{gathered}
$$

where the first term on the right hand side is calculated as

$$
\begin{gathered}
\int_{0}^{\infty} \int_{0}^{\infty} x \mathbb{E}\left[\sum_{i^{\prime} \in \mathcal{M}_{2, i}^{\mathrm{L}}} S_{2, i^{\prime}} \mid X_{1, i}=x, T_{1, i-1}=t, E_{1, i}^{\mathrm{L}}\right] \cdots \\
f_{X_{1, i} T_{1, i-1} \mid E_{1, i}^{\mathrm{L}}}(x, t) \mathrm{d} x \mathrm{~d} t \stackrel{(a)}{=} \frac{1}{\mu} \int_{0}^{\infty} \int_{0}^{\infty} x \mathbb{E}\left[M_{2, i}^{\mathrm{L}} \mid \cdots\right. \\
\left.X_{1, i}=x, T_{1, i-1}=t, E_{1, i}^{\mathrm{L}}\right] f_{X_{1, i} T_{1, i-1} \mid E_{1, i}^{\mathrm{L}}}(x, t) \mathrm{d} x \mathrm{~d} t \\
=\frac{1}{\mu} \int_{0}^{\infty} \int_{0}^{\infty} x \sum_{m=0}^{\infty} m \operatorname{Pr}\left[M_{2, i}^{\mathrm{L}}=m \mid X_{1, i}=x, \cdots\right. \\
\left.T_{1, i-1}=t, E_{1, i}^{\mathrm{L}}\right] f_{X_{1, i} T_{1, i-1} \mid E_{1, i}^{\mathrm{L}}}(x, t) \mathrm{d} x \mathrm{~d} t,
\end{gathered}
$$

where equality (a) follows because the service time $S_{2, i^{\prime}}$ is independent of all other random variables in the system.

The second term on the right hand side of (23) and the final expression in (24) reveal two critical issues in deriving the third conditional expectation term of (8). The second term on the right hand side of (23) contains the possible residual service time of the packet of source 2 that is under service at the arrival instant of packet $1, i, R_{2, i}^{\mathrm{L}}$, which cannot be further simplified. In the final expression in (23), computing the probability $\operatorname{Pr}\left[M_{2, i}^{\mathrm{L}}=m \mid X_{1, i}=x, T_{1, i-1}=t, E_{1, i}^{\mathrm{L}}\right]$ requires complicated transient analysis, which for an $\mathrm{M} / \mathrm{G} / 1$ queueing model is intractable. Thus, we next propose an approximation for (23) and derive an approximate expression for the average AoI in a multi-source M/G/1 queueing model.

To approximate (23), we make the following two simpli- fications for the long event $E_{1, i}^{\mathrm{L}}: 1$ ) we neglect the possible residual service time of source 2 packet that is under service at the arrival instant of packet $1, i$ and 2) we assume that the average number of packets of source 2 that must be served before packet $1, i$ is equal to the average number of packets of source 2 that are queued during the system time of packet $1, i-$ 1. Thus, we assume $\mathbb{E}\left[M_{2, i}^{\mathrm{L}} \mid X_{1, i}=x, T_{1, i-1}=t, E_{1, i}^{\mathrm{L}}\right]=$ $\mathbb{E}\left[J_{2, i}^{\mathrm{L}} \mid X_{1, i}=x, T_{1, i-1}=t, E_{1, i}^{\mathrm{L}}\right]$ in step $(a)$ of (24), where the random variable $J_{2, i}^{\mathrm{L}}$ represents the number of source 2 packets in the system at the departure instant of packet $1, i-1$ for the long event $E_{1, i}^{\mathrm{L}}$. Thus, we assume that during the time interval $x-t$, the arrivals and departures of source 2 packets cancel out. Note that in general, the exact characterization of the number of source 2 packets during the time interval $x-t$ relies on the transient analysis of an M/G/1 queue which is intractable.

With the simplifications above, (23) is approximated as

$$
\begin{aligned}
& \mathbb{E}\left[\left(S_{1, i}^{\mathrm{L}}+R_{2, i}^{\mathrm{L}}\right) X_{1, i} \mid E_{1, i}^{\mathrm{L}}\right] \approx \frac{1}{\mu} \int_{0}^{\infty} \int_{0}^{\infty} x \mathbb{E}\left[J_{2, i}^{\mathrm{L}} \mid X_{1, i}=x, \cdots\right. \\
& \left.T_{1, i-1}=t, E_{1, i}^{\mathrm{L}}\right] f_{X_{1, i}, T_{1, i-1} \mid E_{1, i}^{\mathrm{L}}}(x, t) \mathrm{d} x \mathrm{~d} t \\
& \stackrel{(a)}{=} \rho_{2} \int_{0}^{\infty} \int_{0}^{\infty} t x f_{X_{1, i}, T_{1, i-1} \mid E_{1, i}^{\mathrm{L}}}(x, t) \mathrm{d} x \mathrm{~d} t \\
& \stackrel{(b)}{=} \frac{\rho_{2}}{P\left(E_{1, i}^{\mathrm{L}}\right)} \int_{0}^{\infty} \int_{t}^{\infty} x t \lambda_{1} e^{-\lambda_{1} x} f_{T_{1, i-1}}(t) \mathrm{d} x \mathrm{~d} t \\
& =\frac{\rho_{2}}{P\left(E_{1, i}^{\mathrm{L}}\right)} \int_{0}^{\infty}\left(t^{2} e^{-\lambda_{1} t} f_{T_{1, i-1}}(t)+\frac{t e^{-\lambda_{1} t}}{\lambda_{1}} f_{T_{1, i-1}}(t)\right) \mathrm{d} t \\
& \stackrel{(c)}{=} \frac{\rho_{2}}{P\left(E_{1, i}^{\mathrm{L}}\right)}\left(H_{S}^{\prime \prime}\left(\lambda_{1}\right)-\frac{H_{S}^{\prime}\left(\lambda_{1}\right)}{\lambda_{1}}\right) .
\end{aligned}
$$

where $(a)$ comes from the fact that $\mathbb{E}\left[J_{2, i}^{\mathrm{L}} \mid X_{1, i}=x, T_{1, i-1}=t, E_{1, i}^{\mathrm{L}}\right]=\lambda_{2} t,(b)$ follows from Lemma 5 , and (c) follows from (16).

Lemma 5. The PDF $f_{X_{1, i}, T_{1, i-1} \mid E_{1, i}^{\mathrm{L}}}(x, t)$ is given by

$f_{X_{1, i}, T_{1, i-1} \mid E_{1, i}^{\mathrm{L}}}(x, t)=\frac{1}{P\left(E_{1, i}^{\mathrm{L}}\right)} \begin{cases}0 & x<t \\ \lambda_{1} e^{-\lambda_{1} x} f_{T_{1, i-1}}(t) & x \geq t .\end{cases}$

Due to the space limitations, the proof is given in [14].

By substituting the probabilities $P\left(E_{1, i}^{\mathrm{B}}\right)$ and $P\left(E_{1, i}^{\mathrm{L}}\right)$ given by Lemma 1 and the three derived conditional expectation terms (21), (22), and (25) into (8), an approximation for $\mathbb{E}\left[X_{1, i} W_{1, i}\right]$ is calculated. Finally, by substituting the approximate expression for $\mathbb{E}\left[X_{1, i} W_{1, i}\right]$ and (2) into (1), an approximation for the average AoI of source 1 in a multisource $\mathrm{M} / \mathrm{G} / 1$ queueing model is given as

$$
\begin{aligned}
\Delta_{1} & \approx \mathbb{E}[W]+\frac{2}{\mu}+\frac{2 H_{S}\left(\lambda_{1}\right)}{\lambda_{1}}-H_{S}^{\prime}\left(\lambda_{1}\right) \\
& -\frac{1}{\lambda_{1}}+\rho_{2}\left(\frac{2}{\lambda_{1}}+H_{S}^{\prime}\left(\lambda_{1}\right)-\frac{2 H_{S}\left(\lambda_{1}\right)}{\lambda_{1}}\right),
\end{aligned}
$$

where the average waiting time of each packet in the system, $\mathbb{E}[W]$, is given as $\mathbb{E}[W]=\mathbb{E}\left[S^{2}\right] \lambda /(2-2 \rho)$ [16, Sect. 3], where $\mathbb{E}\left[S^{2}\right]=2 / \mu^{2}$ is the second moment of the service time, 


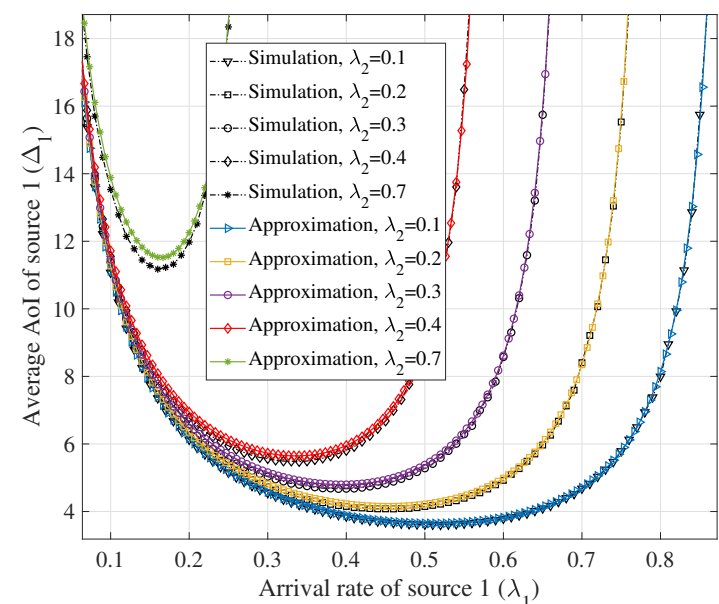

Fig. 1: The average AoI of source 1 as a function of $\lambda_{1}$ for different values of $\lambda_{2}$ with $\mathbb{E}[S]=1, \kappa=2$, and $\beta_{2}=2$.

$H_{S}\left(\lambda_{1}\right)$ is a function of the Laplace transform of the PDF of the service time calculated by (12), and $H_{S}^{\prime}\left(\lambda_{1}\right)$ and $H_{S}^{\prime \prime}\left(\lambda_{1}\right)$ are the first and second derivative of $H_{S}(\cdot)$ at $\lambda_{1}$.

\section{VAlidation And Simulation Results}

We evaluate the accuracy of the proposed approximate expression in (26) in the considered queueing model where the service time follows a gamma distribution. A gamma distribution can be used to characterize the service time in a wireless system where a transmitter is not directly accessible to its intended receiver. Consider a relay network with multiple wireless hops between a transmitter and the receiver. If the service time of each hop follows an exponential distribution, the end-to-end service time in the system follows a gamma distribution [17]. The PDF of a gamma distributed random variable $S$ is defined as $f_{S}(s)=\operatorname{Gamma}(s ; \kappa, \beta)=\frac{\beta^{\kappa} s^{\kappa-1} \exp (-\beta s)}{\Gamma(\kappa)}, s>0$, with parameters $\kappa>0$ and $\beta>0$, where $\Gamma(\kappa)$ is the gamma function at $\kappa$. The first and the second moment of this random variable is $\mathbb{E}[S]=\kappa / \beta$ and $\mathbb{E}\left[S^{2}\right]=\kappa / \beta^{2}$, respectively. A gamma distribution with parameter $\kappa$ and $\beta$ can be interpreted as the summation of $\kappa$ independent exponential random variables with parameter $\beta$.

Fig. 1 shows the average AoI of source 1 as a function of $\lambda_{1}$ for different values of $\lambda_{2}$ with a fixed mean service time of the system $\mathbb{E}[S]=1, \kappa=2$, and $\beta=2$. The figure shows that the derived approximate expression in (26) provides an accurate estimate of the average AoI in the considered queueing model where the service time follows a gamma distribution.

\section{CONCLUSions}

We considered a single-server multi-source FCFS queueing model with Poisson arrivals and analyzed the average AoI of each source. We derived an approximate expression for the average AoI for a multi-source M/G/1 queueing model. The simulation results showed that the approximate expression for the average AoI is sufficiently accurate for a gamma distributed service time.

\section{ACKNOWLEDGEMENTS}

The authors would like to thank Prof. Roy Yates for providing invaluable guidance toward characterizing the AoI in the considered queueing model.

This research has been financially supported by the Infotech Oulu, the Academy of Finland (grant 323698), and Academy of Finland 6Genesis Flagship (grant 318927). M. Codreanu would like to acknowledge the support of the European Union's Horizon 2020 research and innovation programme under the Marie Skłodowska-Curie Grant Agreement No. 793402 (COMPRESS NETS). The work of M. Leinonen has also been financially supported in part by the Academy of Finland (grant 319485). M. Moltafet would like to acknowledge the support of Finnish Foundation for Technology Promotion.

\section{REFERENCES}

[1] P. Corke, T. Wark, R. Jurdak, W. Hu, P. Valencia, and D. Moore, "Environmental wireless sensor networks," Proc. IEEE, vol. 98, no. 11, pp. 1903-1917, Nov. 2010.

[2] R. D. Yates and S. K. Kaul, "The age of information: Real-time status updating by multiple sources," IEEE Trans. Inform. Theory, vol. 65, no. 3, pp. 1807-1827, Mar. 2019.

[3] M. Moltafet, M. Leinonen, M. Codreanu, and N. Pappas, "Power minimization in wireless sensor networks with constrained AoI using stochastic optimization," in Proc. Annual Asilomar Conf. Signals, Syst., Comp., Pacific Grove, USA, Nov. 3-6, 2019. [Online]. https://arxiv.org/pdf/1912.02421v1.

[4] M. Moltafet, M. Leinonen, and M. Codreanu, "Worst case age of information in wireless sensor networks: A multi-access channel," IEEE Wireless Commun. Lett., Early Access 2019.

[5] - "Worst case analysis of age of information in a shared-access channel," in Proc. Int. Symp. Wireless Commun. Systems, Oulu, Finland, Aug. 27-30, 2019, pp. 613-617.

[6] P. Papadimitratos, A. D. L. Fortelle, K. Evenssen, R. Brignolo, and S. Cosenza, "Vehicular communication systems: Enabling technologies, applications, and future outlook on intelligent transportation," IEEE Commun. Mag., vol. 47, no. 11, pp. 84-95, Nov. 2009.

[7] S. Kaul, R. Yates, and M. Gruteser, "Real-time status: How often should one update?" in Proc. IEEE Int. Conf. on Computer. Commun. (INFOCOM), Orlando, FL, USA, Mar. 25-30, 2012, pp. 2731-2735.

[8] S. K. Kaul, R. D. Yates, and M. Gruteser, "Status updates through queues," in Proc. Conf. Inform. Sciences Syst. (CISS), Princeton, NJ, USA, Mar. 21-23, 2012, pp. 1-6.

[9] R. D. Yates and S. Kaul, "Real-time status updating: Multiple sources," in Proc. IEEE Int. Symp. Inform. Theory, Cambridge, MA, USA, Jul. 1-6, 2012, pp. 2666-2670.

[10] E. Najm and E. Telatar, "Status updates in a multi-stream M/G/1/1 preemptive queue," in Proc. IEEE Int. Conf. on Computer. Commun. (INFOCOM), Honolulu, HI, USA, Apr. 15-19, 2018, pp. 124-129.

[11] M. Moltafet, M. Leinonen, and M. Codreanu, "Closed-form expression for the average age of information in a multi-source M/G/1 queueing model," in Proc. IEEE Inform. Theory Workshop, Visby, Gotland, Sweden, Aug. 25-28, 2019.

[12] A. Kosta, N. Pappas, and V. Angelakis, "Age of information: A new concept, metric, and tool," Foun. and Trends in Net., vol. 12, no. 3, pp. 162-259, 2017.

[13] J. N. Daigle, Queueing Theory with Applications to Packet Telecommunication. New York: Springer Science, 2005.

[14] M. Moltafet, M. Leinonen, and M. Codreanu, "On the age of information in multi-source queueing models," Submitted to IEEE Trans. Commun, [Online]. https://arxiv.org/abs/1911.07029v1, 2019.

[15] L. Rade and B. Westergren, Mathematics Handbook for Science and Engineering. Berlin, Germany: Springer, 2005.

[16] D. Bertsekas and R. Gallager, Data Networks. Englewood Cliffs, New Jersey: Prentice-Hall International, 1992.

[17] E. Najm and R. Nasser, "Age of information: The gamma awakening," in Proc. IEEE Int. Symp. Inform. Theory, Barcelona, Spain, Jul. 10-16, 2016, pp. 2574-2578. 